\title{
Zukunftsperspektiven der Ökobilanzierung
}

\author{
Tobias Viere ${ }^{1} \cdot$ Nadine Rötzer $^{1}$
}

Online publiziert: 12. Mai 2016

(c) Springer-Verlag Berlin Heidelberg 2016

Steigende Abfallprobleme, Energieengpässe und die allgemeine Erkenntnis, dass die Ressourcen unserer Erde endlich sind, brachten in den 1970er-Jahren die ersten Ökobilanzen hervor. Seitdem werden ökobilanzielle Methoden in vielen Zusammenhängen eingesetzt, die von der Etikettierung von Produkten mit $\mathrm{CO}_{2}$-Labeln für Marketingzwecke bis zu komplexen wissenschaftlichen Hintergrundanalysen für politische Entscheidungsprozesse reichen. Weltweit hat die Ökobilanzierung in den letzten Jahren wieder stark an Bedeutung gewonnen, was sich auf vielfältige Weise äußert. $\mathrm{Zu}$ nennen sind hier u. a. der Bedeutungsgewinn von Carbon und Water Footprints, die Standardisierung von Ökobilanzergebnissen in Umweltproduktdeklarationen oder die zunehmende Bedeutung lebenswegbezogener Informationen in Umwelt- und Nachhaltigkeitsberichterstattung und in entsprechenden Managementsystemen.

In dieser Ausgabe des UmweltWirtschaftsForum (UWF) wird ein Blick in die Zukunft der Ökobilanzierung im deutschsprachigen Raum gewagt. Dazu haben wir insbesondere NachwuchswissenschaftlerInnen aufgefordert, uns Beiträge zu senden, die ihre ökobilanziellen Forschungsvorhaben und Ergebnisse präsentieren und so erkennen lassen, was im Themenfeld derzeit neu und relevant ist. Eingebettet ist diese Ausgabe in die Tagungsreihe der Ökobilanzwerkstätten, zu denen sich seit über einem Jahrzehnt DoktorandInnen aus dem deutschsprachigen Raum zusammenfinden und ihre Fragen und Erkenntnisse diskutieren.

Tobias Viere

tobias.viere@hs-pforzheim.de

Nadine Rötzer

nadine.roetzer@hs-pforzheim.de

1 Pforzheim, Deutschland
Den Auftakt dieses Hefts macht folgerichtig die Schirmherrin der Ökobilanzwerkstätten, Prof. Dr. Liselotte Schebek von der Technischen Universität Darmstadt, die langfristige Entwicklungen und aktuelle Herausforderungen im Spannungsfeld von Wissenschaft und Praxis zusammenfasst.

Die folgenden Beiträge zeigen die große Bandbreite der aktuellen ökobilanziellen Forschung auf. Dabei fällt auf, dass einige Themen seit Anbeginn der Ökobilanzierung Forschungsgegenstand sind und bleiben. Hier ist insbesondere die Allokationsthematik zu nennen, welche in den Beiträgen von Suski \& Wiesen sowie von Hottenroth et al. behandelt wird. Ein stets präsentes Thema ist der Umgang mit Datenlücken, die Klein \& Stock in ihrem Beitrag für chemische Produkte auf innovative Weise zu schließen versuchen. Auch Energiesysteme gehören seit jeher zum Kern ökobilanzieller Betrachtungen (siehe hierzu Wall sowie Spielmann \& Schebek).

$\mathrm{Zu}$ den häufigen Anwendungen von Ökobilanzen zählen System- und Technologievergleiche, die zunehmend in den frühen Entwicklungsstadien neuer Produkte und Technologien eingesetzt werden, um deren Umweltwirkungen und somit mögliche Vorteile gegenüber vorhandenen Herstellungsverfahren aufzuzeigen (siehe hierzu Hoppe \& Bringezu, Dorn et al. und Clausen \& Pretz). Oft treten dabei nachwachsende Rohstoffe in den Vordergrund, die als Alternative für herkömmliche fossile Rohstoffe in Erwägung gezogen werden und deren tatsächlicher Beitrag zur Schonung des Klimas und der endlichen Ressourcen quantifiziert werden muss. Die Einsatzgebiete von nachwachsenden Rohstoffen reichen dabei von der Energieerzeugung bis hin zur Entwicklung biobasierter Einsatzstoffe oder Produkte (siehe dazu die Beiträge von Spierling et al. sowie Risse \& Richter). Hierzu passt das schon in mehreren UWF-Heften behandelte Thema der Ressourceneffizienz. Wie dieses in Ökobilanzen berücksichtigt wird, wird im Beitrag von 
Thonemann \& Schumann erörtert. Schmidt geht in seinem Beitrag darauf ein, wie beide Aspekte - Ressourceneffizienz und Lebenszyklusanalysen - systematischer Bestandteil von Lehre und anwendungsnaher Forschung werden können. Die Relevanz der Ökobilanzierung für Unternehmen hängt nicht zuletzt auch von den Möglichkeiten zur Integration von finanziellen Entscheidungsgrößen ab, und so stellen Ansätze zur Kombination ökonomischer und ökologischer Analysen ein weiteres wichtiges Zukunftsthema dar (siehe dazu die Beiträge Rieckhof et al. und Meynerts et al.). Zur Frage der Tauglichkeit ökobilanzieller Methoden in der betrieblichen Praxis bietet ferner der Beitrag von Lehmann et al. wichtige Einblicke, der die Bestrebungen der Europäischen Union hinsichtlich einer standardisierten Methodik von Ökobilanzen im Rahmen des Product Environmental Footprint (PEF) analysiert.

Mit der Ausgabe 1/2016 wurden die im UmweltWirtschaftsForum erscheinenden Schwerpunktartikel erstmals auch einem edv-technisch formalisierten externen Begutachtungsverfahren unterzogen, das auf ein hinterlegtes Set von FachexpertInnen zurückgreift, die entsprechend ihrer sachspezifisch angegebenen Kompetenzen eingeschaltet werden. Damit hat sich das UWF auch formal dem international üblichen Fachbegutachtungsprocedere angepasst, um so gerade auch jungen talentierten WissenschaftlerInnen als wertvolles Forum für deren weitere Profilierung dienen zu können. Einige der o.g. NachwuchswissenschaftlerInnen veröffentlichen in einem solchen Kontext zum ersten Mal, und so waren in wenigen Fällen mehrere Rückkopplungsschleifen notwendig, um den damit einhergehenden Mindeststandards an wissenschaftlich-inhaltlicher Qualität und substanziellem Innovationsgehalt in hinreichendem Maße gerecht werden zu können. Besonderer Dank gilt daher den eingeschalteten FachgutachterInnen aus dem ökobilanziellen Umfeld, die mit ihren teilweise recht umfangreichen, kritischen und sehr konstruktiven Anmerkungen und Anregungen wesentlich dazu beigetragen haben, dass die nun vorliegende UWF-Schwerpunktausgabe ,Zukunftsperspektiven der Ökobilanzierung“" in diesem besonderen Anspruch und Facettenreichtum erscheinen konnte. Dank gilt dabei auch den Herausgebern des UmweltWirtschaftsForums, Prof. Dr. Günter Liesegang und Dr. Thomas Sterr, den MitarbeiterInnen des Springer Verlags und insbesondere unserem Kollegen und Mitherausgeber des UWF Prof. Dr. Mario Schmidt, der diese Ausgabe auf vielfältige Weise unterstützt hat.

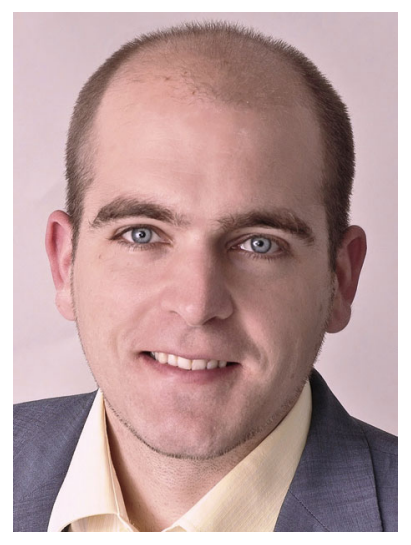

Tobias Viere

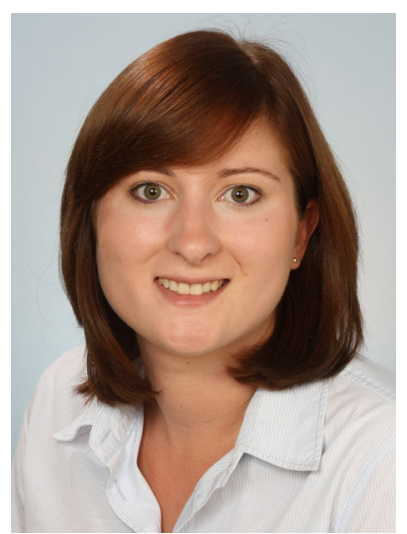

Nadine Rötzer 